\title{
Maladies non transmissibles: combiner les perspectives
}

\author{
Dr méd. Carlos Beat Quinto
}

Membre du Comité central de la FMH, responsable du département Santé publique et professions de la santé

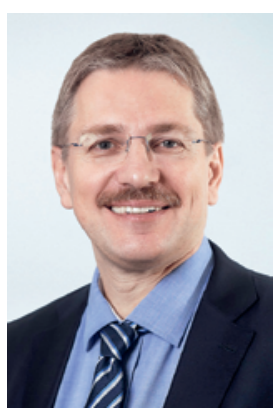

Le présent numéro du Bulletin des médecins suisses vous propose deux courts articles sur l'importance des maladies non transmissibles (MNT). Imaginons que ces dernières soient une immense forêt tropicale. Dans son article centré sur les aspects économiques de la santé, Helsana l'observe en adoptant une perspective générale: de grandes quantités d'informations tirées de bases de données existantes ont été analysées et de nombreuses hypothèses posées. L'article des Instituts universitaires de médecine de famille privilégie en revanche la perspective d'une expédition scientifique: les chercheurs ont analysé un plus petit volume de données, recueillies spécifiquement pour traiter leur problématique, et leurs descriptions sont plus nuancées. Les résultats des deux articles se recoupent en ce qui concerne l'importance des maladies cardiovasculaires et psychiques. Les publications liées au projet de recherche des instituts universitaires présentent non seulement des conclusions intéressantes sur la complexité de la multimorbidité, mais aussi des pistes importantes pour une perspective supplémentaire, celle de la santé publique. En Suisse, les maladies non transmissibles, qui occasionnent $80 \%$ des coûts de la santé, sont principalement dues au tabac. La santé publique permet d'expliquer pourquoi cette forêt progresse ou recule.

Une perspective générale montre que la majeure partie des coûts de la santé sont engendrés par des maladies qui nous touchent tous.

Venons-en aux maladies non transmissibles abordées dans les deux articles. L'augmentation des troubles dépressifs découle-t-elle aussi de la tertiarisation de la société et de l'obligation d'être joignable jour et nuit? Dans la perspective de l'expédition scientifique, il ne faut pas mettre tous les patients «dépressifs» dans le même panier, puisque le traitement, les ressources nécessaires et la procédure varient fortement selon le type de "dépression». Prenons maintenant l'exemple des maux de dos: ils peuvent être entraînés - chez les rares personnes qui exécutent un travail physique pénible - par usure, mais aussi - chez tous ceux qui exercent une activité sédentaire - par le déconditionnement de la musculature dorsale. Et qu'en est-il des maladies cardiovasculaires courantes et de l'augmentation des maladies pulmonaires chroniques? Sontelles l'expression d'une société qui libéralise, avec la bénédiction du Parlement, la publicité pour le tabac s'adressant aux enfants et aux adolescents et se moque des coûts consécutifs, d'un montant de plusieurs milliards? En adoptant une perspective générale, on constate que la majeure partie des coûts de la santé est engendrée par des maladies qui touchent beaucoup de monde, et donc chacun d'entre nous.

De par leur structure, les deux articles laissent cependant subsister quelques zones d'ombre: l'encadrement social des patients atteints de démence occasionne des coûts plus élevés que les soins aigus qui leur sont dispensés. Or, vu que la multimorbidité constitue plutôt la règle que l'exception dans la tranche d'âge où les démences sont prévalentes, une prise en charge purement sociale ne saurait suffire. Des soins infirmiers et médicaux de qualité sont également nécessaires. La démence interagissant avec d'autres maladies, les discussions menées avec le patient et ses proches peuvent modifier l'ordre des priorités ainsi que le traitement d'autres maladies. Ce type de discussion nécessite du temps et repose sur la confiance.

Pour progresser, il faut que les tenants des différentes perspectives discutent ensemble en respectant les points de vue de chacun dans toute leur complexité et en reconnaissant leurs propres limites. Seul un dialogue serein et ouvert permettra de réaliser de vrais progrès. Imposer une perspective unique revient à apporter de l'eau au moulin d'une "société de défiance», qui, basée sur des résultats peu fiables, échouera face à l'inefficacité des contrôles administratifs.

Les maladies non transmissibles ont une importance capitale dans le domaine des soins. L'avenir nous dira si le deuxième paquet de mesures «Prévention dans le domaine des soins» de la stratégie MNT va dans le sens du plan de mesures publié initialement.

Prenez le temps de lire ces deux articles, cela en vaut la peine! 